\title{
Sensory Activation and Role of Inhibitory Reticulospinal Neurons that Stop Swimming in Hatchling Frog Tadpoles
}

\author{
Ray Perrins, Alison Walford, and Alan Roberts \\ School of Biological Sciences, University of Bristol, Bristol, BS8 1UG, United Kingdom
}

Activity in neuronal networks underlying locomotion and other rhythmic actions can start and stop in response to specific sensory stimuli. In vertebrate locomotion, some reticulospinal neurons such as Mauthner neurons can initiate activity, but the neurons controlling stopping are not defined. We have studied swimming in tadpoles of the frog, Xenopus, which is started by touching the skin and stops when the head contacts a solid surface. Using an immobilized tadpole preparation, the same stimuli control fictive swimming. When head contact is imitated by pressure to the head skin sensory neurons in the trigeminal ganglion are active, spinal neurons receive GABAergic inhibition, and swimming stops. Here we record intracellularly from neurons in the hindbrain that are excited by pressure or electrical stimulation to the head skin. By intracellular filling with

Locomotion needs to be started and stopped in response to specific behavioral cues. It is known that specific stimuli can stop locomotion in a range of animals (dogfish: Gray and Sand, 1936; insects: Fraenkel, 1932; Pringle, 1974; molluscs: Satterlie et al., 1985), but the pathways have not been defined at the neuronal level. It is especially difficult to investigate natural stopping pathways in the case of vertebrates. GABAergic reticulospinal neurons have been shown to project to the spinal cord in the rat (Holstege, 1991). In the cat, inhibitory connections have been found between brainstem and spinal neurons (Magoun and Rhines, 1946; Llinas and Terzuolo, 1964). Brainstem stimulation in freely behaving cats is thought to stop walking by exciting spinal inhibitory neurons (Mori, 1987). However, detailed analysis of the pathways requires immobilized preparations where continuous artificial stimulation (either electrical or via excitatory agonists) is generally needed to activate the locomotor central pattern generators (CPGs) (Stein et al., 1997). Such reliance on continual stimulation makes it particularly difficult to study pathways that naturally stop locomotion. In contrast, in immobilized Xenopus tadpoles, fictive swimming activity can be initiated by brief touch and is then maintained for many seconds without further input (Roberts et al., 1997). The tadpole nervous system is relatively simple, and access to the CNS requires minimal dissection (Fig. 1). This means that sensory pathways can be stimulated mechanically or electrically in immobilized preparations.

Received Jan. 9, 2002; revised March 5, 2002; accepted March 6, 2002.

This work was supported by the Wellcome Trust. We thank Derek Dunn, Julie Hansen, Tim Colburn, and Linda Teagle for technical assistance and Steve Soffe for advice and comments on this manuscript.

Correspondence should be addressed to Prof. Alan Roberts, School of Biological Sciences, University of Bristol, Woodland Road, Bristol, BS8 1UG, UK. E-mail: A.Roberts@bristol.ac.uk.

Copyright (C) 2002 Society for Neuroscience $\quad 0270-6474 / 02 / 224229-12 \$ 15.00 / 0$ neurobiotin, we identify these anatomically as mid-hindbrain reticulospinal neurons (MHRs). These have contralateral descending projections to the spinal cord and GABA-like immunoreactivity. They are rhythmically inhibited during fictive swimming. Individual MHRs reliably stopped ongoing swimming when brief firing was induced by intracellular current injection. The ability of individual MHRs to stop swimming was blocked by the $\mathrm{GABA}_{\mathrm{A}}$ antagonist bicuculline. Our evidence indicates that $\mathrm{MHR}$ receive direct excitation from trigeminal sensory neurons and in turn release GABA to directly inhibit spinal neurons and turn off the swimming central pattern generator.

Key words: locomotion; central pattern generator; trigeminal; GABA; Xenopus; brainstem

In Xenopus tadpoles swimming normally stops when the tadpole contacts an object or the water surface with its head (Roberts and Blight, 1975; Roberts et al., 2000). The tadpole then sticks to the surface with mucous secreted by its cement gland (Fig. 1). The head and cement gland are innervated by free nerve endings from primary trigeminal afferent neurons with central axons that project to the caudal hindbrain (Roberts and Blight, 1975; Roberts, 1980; Hayes and Roberts, 1983). Slowly pressing a probe against the head or cement gland excites one class of these trigeminal afferents (Roberts and Blight, 1975; Roberts 1998) and also reliably stops fictive swimming in immobilized Xenopus tadpoles (Boothby and Roberts, 1992a). This stopping response was blocked by the $\mathrm{GABA}_{\mathrm{A}}$ antagonist bicuculline, and when spinal neurons active during swimming were recorded intracellularly, they were found to receive $\mathrm{GABA}_{\mathrm{A}}$-mediated inhibition after a press to the cement gland (Boothby and Roberts, 1992b). This led to the proposal that GABAergic reticulospinal neurons, found in the Xenopus tadpole using GABA immunocytochemistry (Roberts et al., 1987), could inhibit spinal neurons that normally drive swimming. Boothby and Roberts (1992b) therefore made intracellular recordings from the hindbrain to search for neurons excited by pressing the head. Some were found, but their outputs and anatomy were not characterized.

The purpose of this study was to characterize the anatomy and physiology of reticulospinal neurons excited by pressing the head skin and to obtain evidence for their role in stopping swimming. For the first time in any system, we have defined the role of a class of inhibitory reticulospinal neuron.

\section{MATERIALS AND METHODS}

Recordings were made from 78 hatchling tadpoles of Xenopus laevis (developmental stage 37-38; Nieuwkoop and Faber, 1956). Experiments were performed at $18-22^{\circ} \mathrm{C}$ in a saline of composition (in $\mathrm{mM}$ ): $\mathrm{NaCl}$, 


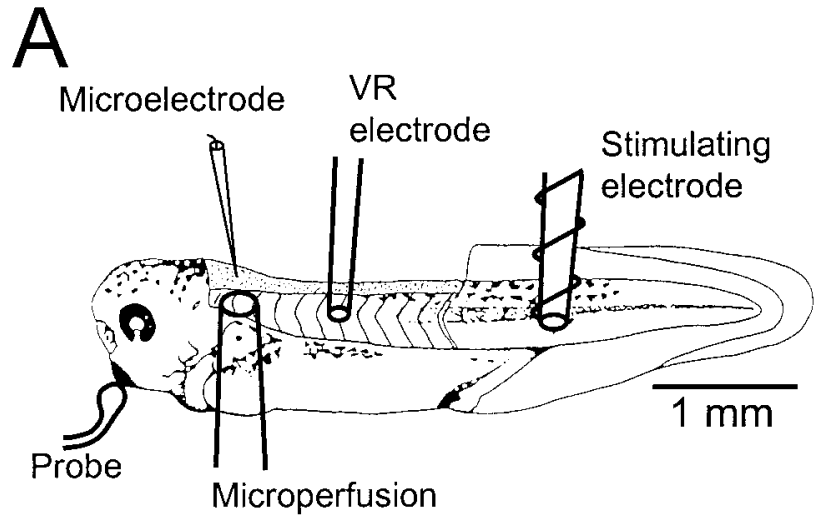

B

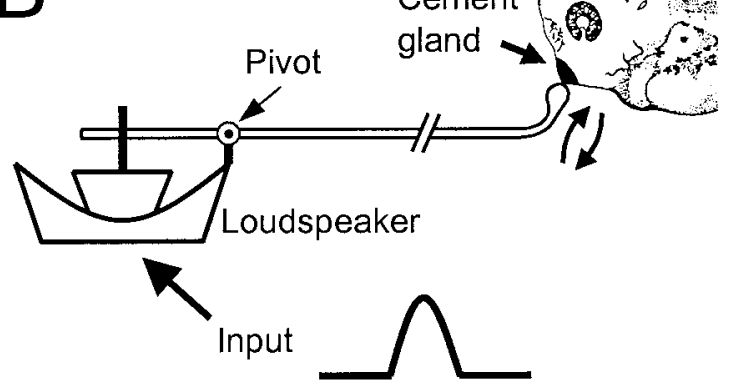

Figure 1. The preparation used for studying hindbrain neurons and their spinal targets. $A$, The immobilized tadpole is pinned on its right side. Swimming is started using a stimulating electrode on the tail skin and motor output monitored using a suction ventral root $(V R)$ electrode. Intracellular recordings can be made from the hindbrain (as illustrated) or the spinal cord. Drugs are applied to neurons via a microperfusion system. Pressure is applied to the head using a glass probe. $B$, The glass probe is attached to a loudspeaker via a lever pivoting about the point indicated. The half-sinusoid shape of the input to the loudspeaker is shown.

115; $\mathrm{KCl}, 3 ; \mathrm{CaCl}_{2}, 4 ; \mathrm{MgCl}_{2}, 1 ; \mathrm{NaHCO}_{3}, 2.4 ; \mathrm{HEPES}, 10$, at $\mathrm{pH}$ 7.4. Animals were anesthetized in a $0.1 \%$ solution of MS-222 (Sigma, St. Louis, MO) in saline and the dorsal fin slit to allow access of the neuromuscular blocker $\alpha$-bungarotoxin $(10 \mu \mathrm{M})$. The tadpoles were left in $\alpha$-bungarotoxin until they no longer swam in response to normally effective stimuli. The tadpoles were then pinned to a rotatable Sylgard (Dow Corning, Wiesbaden, Germany) block in a bath continually perfused with saline (see Fig. 1 for the basic experimental set up). The skin from the otic capsule to the 12th post-otic myotome on the left side of the tadpole was removed with finely etched tungsten microneedles. Extracellular recordings were made from the ventral roots using suction electrodes placed on the intermyotomal clefts exposed by this procedure. The dorsal parts of the myotomes were removed to expose the rostral spinal cord and hindbrain. Intracellular recordings were made by inserting glass microelectrodes into the spinal cord or hindbrain. Fictive swimming was evoked by a $0.5 \mathrm{msec}$ electric current pulse applied to the trunk skin at the level of the anus via a suction electrode. Drugs were then applied via a multibarreled microperfusion system with an opening of $60 \mu \mathrm{m}$ placed $100 \mu \mathrm{m}$ from the CNS.

Microelectrodes with DC resistances of $150-250 \mathrm{M} \Omega$ were pulled on a Sutter P-97 microelectrode puller and filled with $3 \mathrm{M}$ potassium acetate or $2 \mathrm{M}$ potassium acetate with $1 \%$ Neurobiotin (Molecular Probes, Eugene, OR). Neurons were impaled using a Burleigh PZ-100 piezoelectric step driver and using capacitance overcompensation. Neurons were used if they had a stable resting potential more negative than $-50 \mathrm{mV}$ and showed clear rhythmic synaptic input during fictive swimming (Boothby and Roberts, 1992b).

After testing their physiological responses, neurobiotin (Vector Laboratories, Burlingame, CA) was injected into the neurons using positive $0.5 \mathrm{sec}$ duration current pulses of $0.1-0.3 \mathrm{nA}$ applied once per second for
5-10 min. The electrode was then withdrawn, and the animals were left for 30-60 $\mathrm{min}$ for the dye to spread throughout the neuron, before being fixed in $2 \%$ gluteraldehyde in phosphate buffer (PB; $0.08 \mathrm{M} \mathrm{Na}_{2} \mathrm{HPO}_{4}$ and $0.02 \mathrm{M} \mathrm{NaH}_{2} \mathrm{PO}_{4}, \mathrm{pH} 7.4$ ) for $2 \mathrm{hr}$ at room temperature. After fixation the dorsal parts of the axial muscles were removed from the right side of the animal to facilitate later processing. The preparation was washed in PBS (120 mM NaCl in phosphate buffer, $\mathrm{pH}$ 7.4) for $1 \mathrm{hr}$ with two changes of solution. The preparation was then washed in PBS containing 1\% Triton X-100 for $1 \mathrm{hr}$ with two changes of solution and then incubated for $3 \mathrm{hr}$ in a solution of PBS containing $0.5 \%$ Triton and a 1:200 dilution of ExtrAvidin (Sigma). After washing with three changes of PBS for $1 \mathrm{hr}$, the preparation was placed in $0.05 \%$ diaminobenzidine (DAB) in PBS for $5 \mathrm{~min}$, and then into a solution of $0.05 \mathrm{DAB} 0.03 \%$ hydrogen peroxide for 2-5 $\mathrm{min}$. When the neurons became visible, the reaction was stopped by transferring to PBS. The CNS, with notochord and ventral axial muscles attached, was then dissected out of the preparation and dehydrated, cleared in methyl benzoate and xylene, and mounted on its side in DePeX between coverslips so that it could be examined from both sides. Neurons were drawn using a camera lucida and photographed using a Nikon Coolpix 990 digital camera. Because the mid-hindbrain reticulospinal neurons (MHRs) filled had a contralateral axon, photos were taken at several different focal planes, and then the images were processed and stacked using Adobe Photoshop software. Because specimens shrink by $\sim 22 \%$ during dehydration (Li et al., 2001), we have compensated for this shrinkage by multiplying all anatomical measures in this paper by 1.28 .

In immobilized animals, the stopping pathway was stimulated by pressing the head skin at the region of the cement gland using a $0.5 \mathrm{~mm}$ diameter rounded glass probe (Fig. 1). The probe was attached, via a lever, to a loudspeaker (Roberts and Blight, 1975), which was driven by a sinusoidal voltage generated by Signal software and a Cambridge Electronics Design (Cambridge, UK) 1401 computer interface. The monitor trace on records represents the output voltage to the speaker, rather than directly measuring probe movement. At rest the probe was lightly touching the skin of the cement gland. A downward deflection of the trace represents the probe pressing inwards into the cement gland.

Data were stored using Signal software in conjunction with a Cambridge Electronic Design 1401 computer interface. Current was injected into neurons via the recording electrode. All measures are mean $\pm \mathrm{SD}$.

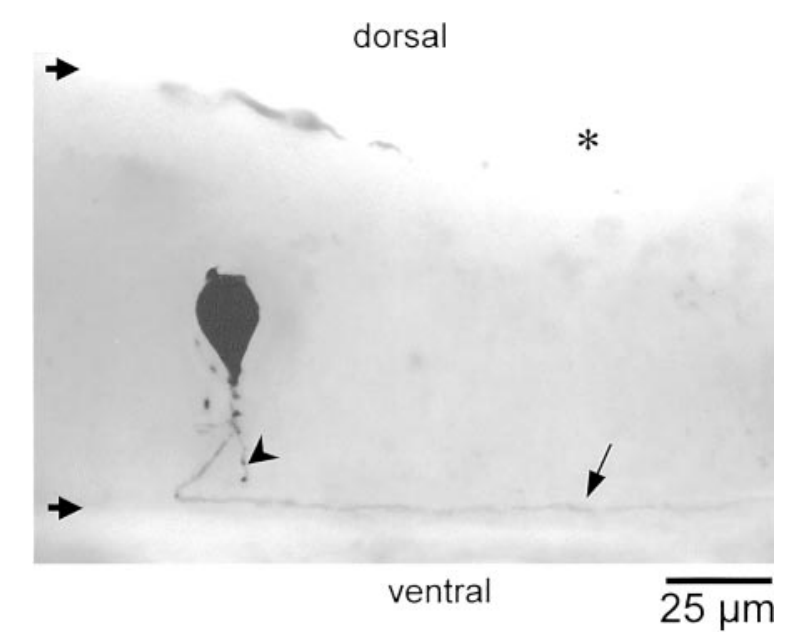

Figure 2. Photomontage of an MHR neuron filled with neurobiotin and also shown in Figure $3, A$ and $D$. Part of the hindbrain near its border with the spinal cord $\left({ }^{*}\right)$ is shown viewed from the left side in a whole mount of the CNS. Rostral is to the left. The pear-shaped MHR soma has a ventral principal process with dendrites (e.g., relatively long ventral dendrite at arrowhead) and an axon that crosses ventrally and then descends on the opposite side (arrow). Short, thick arrows indicate the dorsal and ventral edges of the hindbrain. 

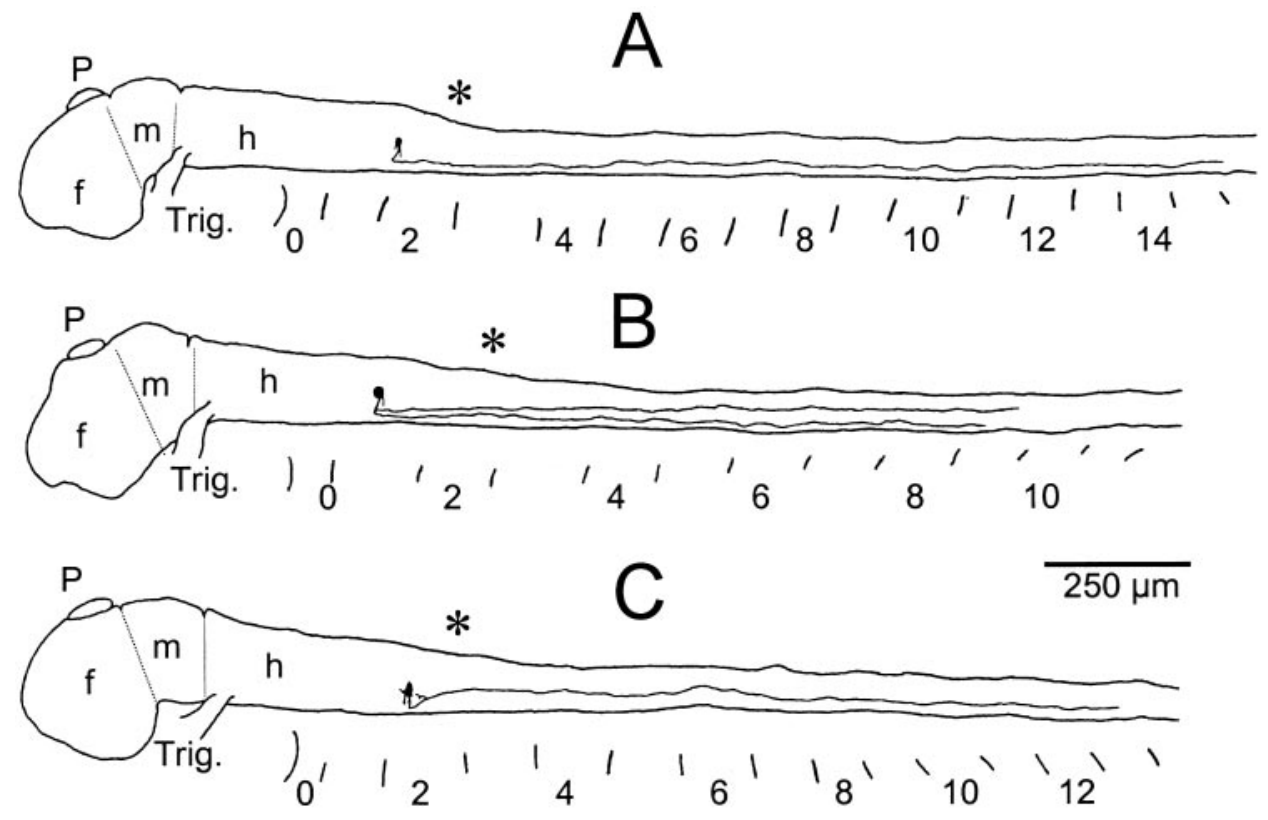

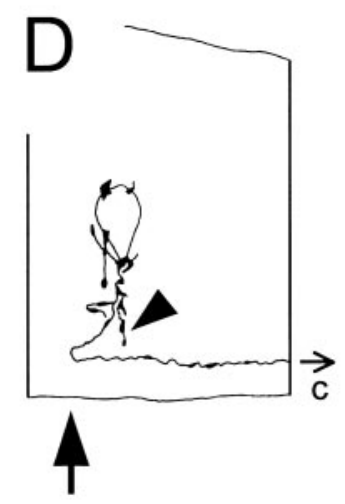

Drugs used: $\alpha$-bungarotoxin $10 \mu \mathrm{M}$, strychnine sulfate $1 \mu \mathrm{M}$, bicuculline methiodide 10-20 $\mu \mathrm{M}$, kynurenate $1 \mathrm{~mm}$ (all Sigma).

\section{RESULTS}

\section{Characterization of hindbrain neurons responding to pressing the cement gland}

Morphology

Ten neurons that responded to pressing the cement gland (see below) were filled successf ully with neurobiotin. Figure 2 shows a composite photograph of a typical filled neuron. Figure 3 shows drawings of three examples at both low and high power, whereas Figure 4 shows all 10 neurons schematically. In a further two cases partial fills were achieved (with only cell body and part of the initial axon segment visible). The cell bodies of the neurons were situated near the dorsoventral midline, in the caudal hindbrain, in the region adjacent to the first and second post-otic myotomes (400-550 $\mu \mathrm{m}$ from the midbrain, approximately equivalent to the sixth to eighth rhombomeres) (Fig. 3). The somata were dorsoventrally elongated (mean height, $28 \pm 3 \mu \mathrm{m}$; mean width, $17 \pm 4 \mu \mathrm{m} ; n=9$ ). All neurons were multipolar, usually with a few, short dorsally projecting dendrites and several elongated, ventral dendrites. All neurons had a contralateral, descending axon between 800- and 1700- $\mu$ m-long (mean, $1180 \pm$ $350 \mu \mathrm{m} ; n=8$ ) (Fig. 4). In addition to this, two examples had

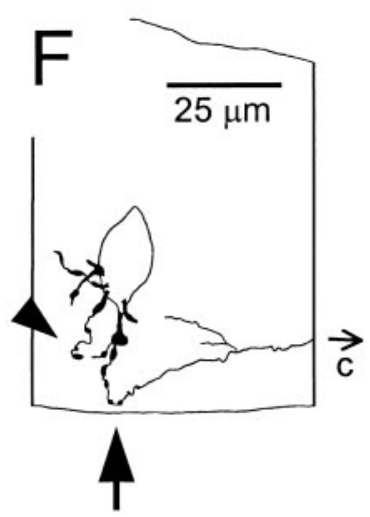

Figure 3. Anatomy of MHR neurons. $A-C$, Scale drawings of a lateral view of the CNS showing the overall anatomy of three neurobiotin filled MHRs. $P$, Pineal gland; Trig, trigeminal nerve; $f$, forebrain; $m$, midbrain; $h$, hindbrain. Asterisk indicates border of hindbrain and spinal cord. Post-otic myotomes are numbered. $D-F$, The region of the soma in lateral view of the same three neurons. Rostral is to the left. Note the relatively long ventral dendrites (arrowheads) and crossed, descending axon. Arrows indicate the point where the axon crosses the ventral midline of the CNS. $c$, Contralateral axon; $i$, ipsilateral axon. ipsilateral descending axons (1140 and $1240 \mu \mathrm{m}$ long) and two had shorter contralateral ascending axons (20 and $280 \mu \mathrm{m}$ in length). Some lengths may be underestimates where axons did not show a distinct end-bulb and may therefore be incompletely filled.

The neurons share the following features with GABAimmunopositive MHRs, as described in Roberts et al. (1987): (1) somata in the hindbrain, $350-550 \mu \mathrm{m}$ from the midbrain-hindbrain border and around the dorsoventral midline, (2) dorsoventrally elongated soma, (3) multipolar soma, with mainly ventrally projecting dendrites, and (4) a ventral principal process that crosses to the contralateral side of the hindbrain then descends into the spinal cord, at least some with an additional ipsilateral descending axon. For simplicity we will now refer to these cells as MHRs.

\section{Neuronal properties}

The mean resting potential for MHRs was $-68 \pm 3 \mathrm{mV}(n=12)$ with an input resistance of $262 \pm 78 \mathrm{M} \Omega(n=12)$. The firing properties of MHR neurons were tested by injecting depolarizing current pulses of $100 \mathrm{msec}$ duration in $0.05 \mathrm{nA}$ steps through the recording electrode (Fig. $5 A, B$ ). The threshold current for the firing was $0.17 \pm 0.06 \mathrm{nA}(n=8)$, and the threshold voltage above the resting potential was $21.6 \pm 3.8 \mathrm{mV}(n=8)$. In response to suprathreshold current, MHR neurons fired multiple action po- 
Figure 4. Schematic representation of the 10 MHR neurons filled by intracellular injection of neurobiotin. The top neuron is superimposed on a diagram of the CNS (viewed dorsally) showing hindbrain, spinal cord, trigeminal nerve (Trig.), otic capsule and numbered myotomes. The remaining neurons are shown to the same scale, and the distance in millimeters from the midhindbrain boundary is shown at the bottom. A dashed line shows the nominal boundary between the hindbrain and spinal cord. Most axons faded at their distal end, but four ended in growth cones, shown by small, black rectangles. Note the limited rostrocaudal distribution of somata.

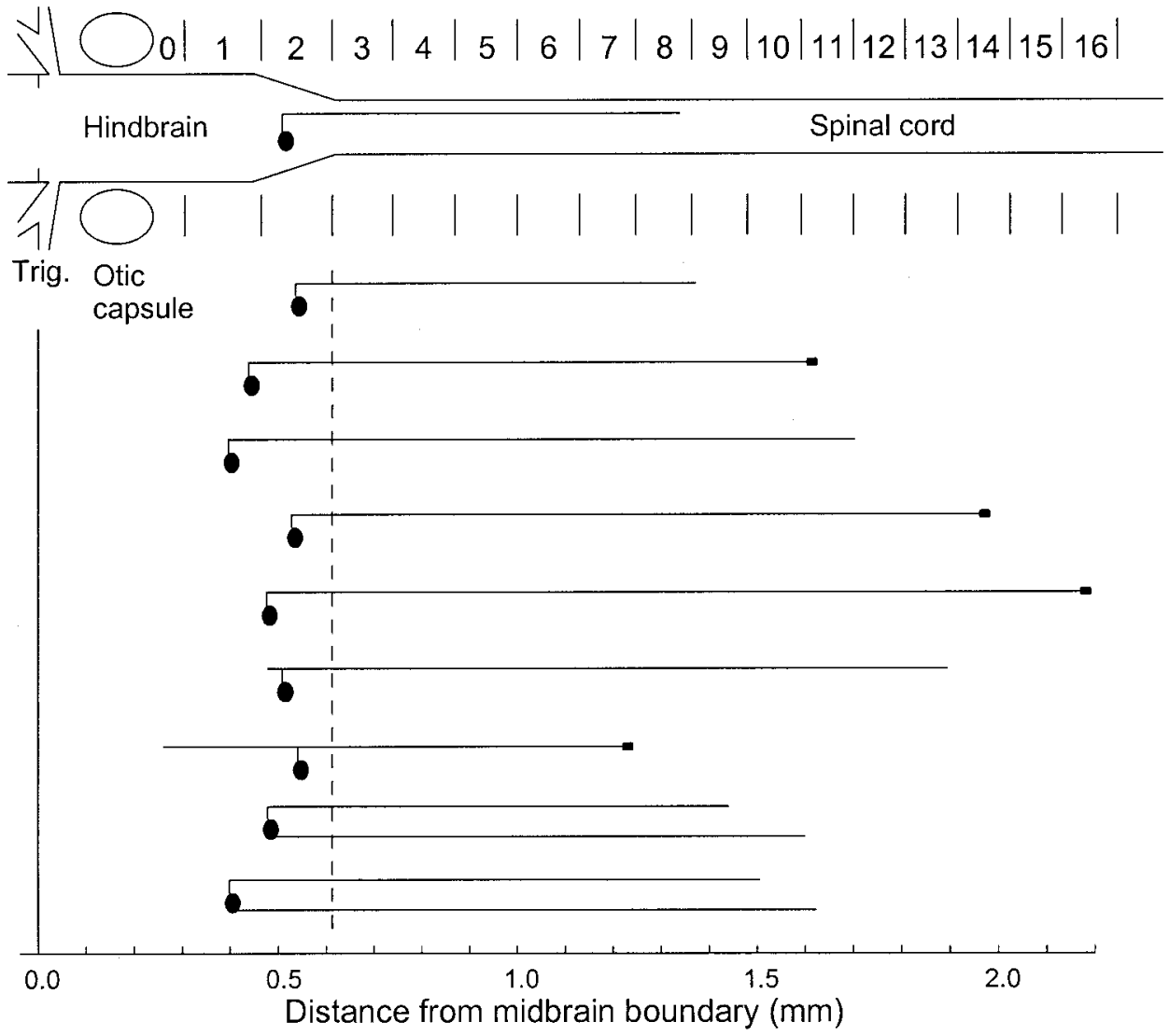

tentials. This is similar to sensory interneurons in the Xenopus spinal cord (Roberts and Sillar, 1990) but is in contrast with motoneurons which usually only fire one action potential, regardless of current (Soffe, 1990; Soffe and Perrins, 1997). Firing frequency was measured as instantaneous firing frequency (from the first two spikes in a train) and over the whole current pulse. Both these measures increased with higher current levels and could be fitted with a single exponential (Fig. 5C). Firing frequency also adapted with time during these current pulses (Fig. 5) $(n=8)$.

\section{Responses of MHRs to activation of pathways that start fictive swimming}

We investigated the responses of MHRs to two different types of input that can start swimming in Xenopus tadpoles. The first of these involves the pineal eye, which is excited by light dimming and can initiate swimming (Foster and Roberts, 1982; Jamieson and Roberts, 2000). Dimming the illumination produced no short- or long-latency EPSPs in the MHRs. The only input was the midcycle glycinergic IPSPs seen once swimming activity started (Fig. 6).

The second type of input investigated was electrical stimulation of the trunk skin, which stimulates the touch pathway to initiate swimming. The stimulating electrode was placed on the skin at the level of the anus, and after a $0.5 \mathrm{msec}$ stimulus above the threshold for swimming, several distinct types of input to MHRs were observed (Fig. 7A,C): (1) An EPSP with a short, fixed latency. This was revealed more clearly when glycinergic inhibition was blocked by strychnine (Fig. 7C). The overall latency of this EPSP was $10.8 \pm 1.6 \mathrm{msec}(n=81$ EPSPs from five prepa- rations). However, the variance of the delay in each preparation was very small $(\sim 0.1 \mathrm{msec})$ (Table 1$)$ and was consistent with a monosynaptic connection (see Discussion). In 2 of 12 cases there were also indications of a short-latency IPSP remaining in strychnine, indicated by a sharp cutoff to the EPSP and the membrane potential going below the resting potential (seen in Fig. $7 A$ but not in $7 C$ ). Because this strychnine-resistant IPSP was not a reliable input, it was not investigated further. (2) EPSPs with long, variable latencies. These were observed in seven of eight neurons tested. The EPSPs occurred during swimming between $100 \mathrm{msec}$ and $1 \mathrm{sec}$ after the stimulus. They occurred at all phases of swimming, apparently at random (compared with ipsilateral ventral root discharge) (Fig. $7 B$ ). The total number of these EPSPs did seem to increase with increasing stimulus intensity, but we have not investigated these EPSPs further in this study. (3) Reliable, midcycle IPSPs. These were seen throughout swimming episodes (Figs. 6, 7A). The IPSPs were only seen at midcycle, compared with ipsilateral ventral root discharge, and were reversibly blocked by the glycine antagonist strychnine $(1 \mu \mathrm{M} ; n=6)$ (Fig. 7A). The midcycle IPSPs were very reliable, occurring on $100 \%$ of cycles (measured on 100 cycles each from six MHRs). This is in contrast to IPSPs in spinal sensory dorsolateral commissural interneurons that tend to fail on some cycles as swimming progresses (Sillar and Roberts, 1992).

\section{Responses of MHRs on activation of the stopping pathway}

When the head skin was pressed to stop fictive swimming, MHRs received excitation and usually fired one or more action potentials (Fig. 8). MHRs also received excitation (a barrage of EPSPs) 

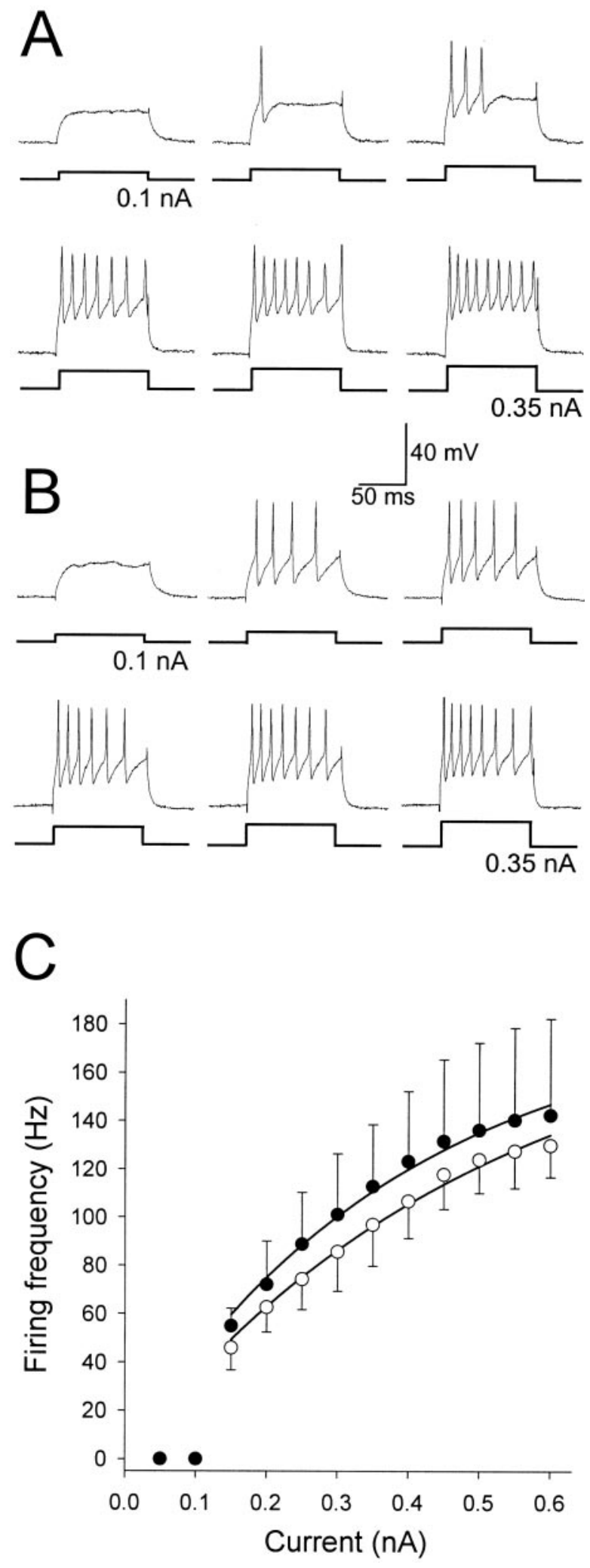

Figure 5. Firing properties of MHRs. $A, B$, Firing responses of two neurons to current pulses from 0.1 to $0.35 \mathrm{nA}$ in $0.05 \mathrm{nA}$ steps. $C$, Graph showing firing frequency against injected current for eight MHRs. Closed circles are instantaneous firing frequency for the first two spikes. Open circles are frequency measured over the whole $100 \mathrm{msec}$ current pulse. Both are fitted with exponential curves.
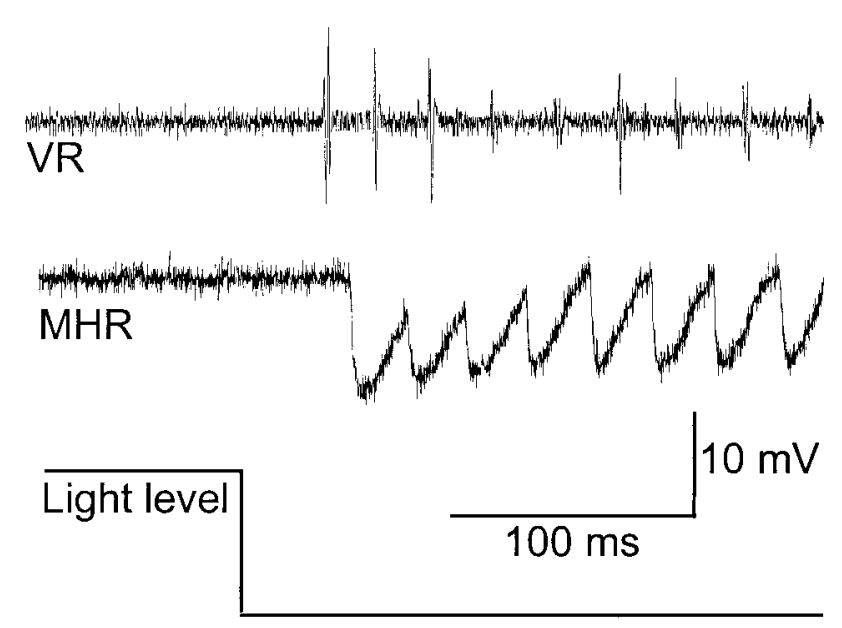

Figure 6. Response of MHR to light dimming over pineal eye. When the light level drops, swimming is initiated and recorded as rhythmic ventral root $(V R)$ bursts. In intracellular record from MHR $(M H R)$ no excitation is seen, but midcycle IPSPs are present on every cycle during swimming.

when the cement gland was pressed in the absence of swimming (Fig. $9 A$ ). The excitation was graded with stimulus intensity $(n=$ 6) (Fig. $9 A$ ), and if the stimulation was strong enough, resulted in firing. Instantaneous firing frequencies in response to pressing the cement gland could reach $>100 \mathrm{~Hz}$, approximately equivalent to $0.35 \mathrm{nA}$ injected current (twice the threshold current) (Fig. 5). The excitation was reversibly blocked by the broad spectrum excitatory amino acid (EAA) antagonist kynurenate $(1 \mathrm{~mm} ; n=$ 6) (Fig. 9A). Repeated pressing of the cement gland resulted in responses that steadily reduced in both duration and amplitude $(n=4)$ (Fig. 9B).

To discover if the MHR neurons were directly excited by trigeminal sensory neurons, we investigated their response to electrical stimulation of the cement gland $(n=5)$ (Fig. 10). In this region of the head there are two types of sensory nerve endings (Roberts, 1980): those that respond to slow pressing and have an inhibitory effect on swimming and those that respond to touch and have excitatory effects. For reasons detailed below we are confident that electrical stimulation using a suction electrode applied to the side of the gland (Fig. 10A) excited afferents sensitive to slow pressing almost exclusively. MHR neurons received EPSPs in response to electrical stimulation, which were graded with stimulus intensity $(n=5)$ (Fig. $10 B)$ and fully blocked by $1 \mathrm{~mm}$ kynurenate $(n=5)$ (Fig. 10C). The delay to these EPSPs was very brief and had low variability within each preparation (see individual variances in Table 1). The mean delay was $5.9 \mathrm{msec} \pm 0.6 \mathrm{SD}(n=25$ EPSPs each from five preparations). The value of the delay and its low variance are consistent with a monosynaptic connection between the trigeminal sensory neurons and the MHR neurons. The delays and their variances are very similar to those found in the known monosynaptic pathway where Rohon-Beard neurons innervating the trunk skin excite spinal sensory interneurons (delay, 6.26 msec $\pm 0.16 ; n=71$ ) (Roberts and Sillar, 1990). In one MHR the EPSPs occasionally gave rise to a spike (Figs. 10B, 11A). The latency to this spike was longer and more variable because of differences in the rise time of the EPSP (mean delay, $12.9 \mathrm{msec} \pm$ 1.9 SD; variance, $1.7 ; n=25$ spikes) (Table 1 ). 

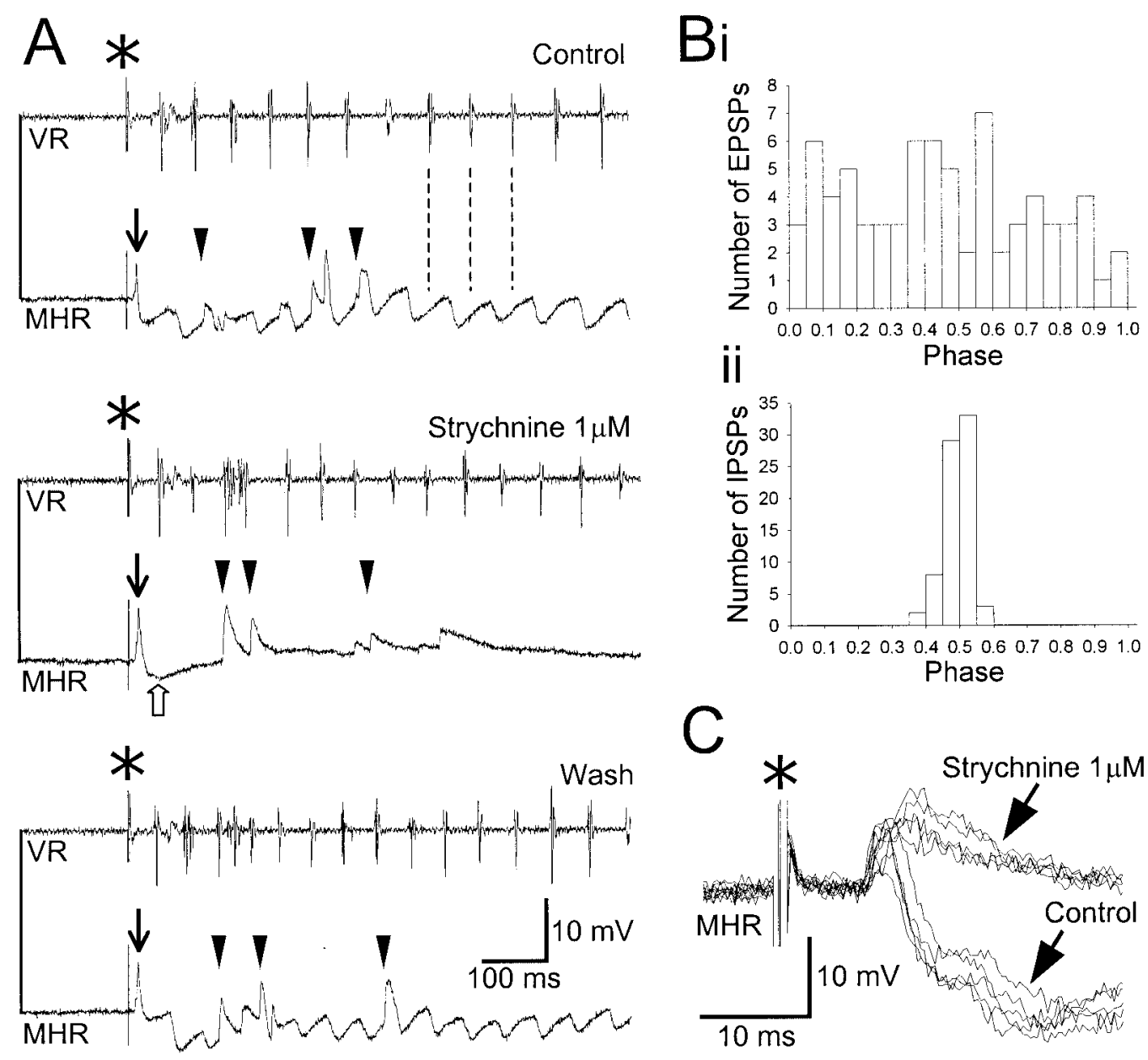

Figure 7. MHR neurons receive excitation after trunk skin stimulation. A, In control, two types of excitation can be seen when the stimulus (at artifact, asterisk) initiates fictive swimming (seen in ventral root record, $V R$ ), a short-latency EPSP (arrows) and longer, variable, latency EPSPs (arrowheads). The inhibition consists of reliable, midcycle IPSPs (dashed lines). Strychnine reversibly blocks the midcycle IPSPs and reveals the excitation more clearly. Note that in strychnine the short-latency EPSP is cut off, and there is a subsequent undershoot below the resting potential, indicating a short-latency IPSP (open arrow). This was only seen in 2 of 12 preparations (compare with $C$, from another preparation). Bi, The number of long-latency EPSPs occurring at different phases of the swimming cycle. No phasic relationship can be seen. Bii, In contrast, the IPSPs during swimming are centered around 0.5 phase (midcycle). $C$, Five traces in control and five in strychnine reveal the short-latency EPSP in response to tail skin stimulation. Note the low variability of the delay to the onset of the EPSP.

\section{Responses of spinal neurons to activation of the stopping pathway}

Because electrical stimulation of the cement gland excited the MHRs and we propose that MHRs inhibit spinal neurons, we also looked at responses to cement gland stimulation in rhythmically active, ventral spinal neurons (presumed motoneurons). In five of six spinal neurons in six separate preparations the response was purely inhibitory (Figs. $10 D, 11 A$ ) and was fully blocked by bicuculline (Fig. 10D). In one case an excitatory response was also observed at a similar latency. This was presumably because of the activation of fast transient (touch) receptors, but in general it appears that electrical stimulation near the cement gland primarily activated the pressure sensitive afferents.

In each preparation tested, the delay to the start of the IPSPs in ventral neurons was longer and the variation larger than for the EPSPs in MHRs (Fig. 11, Table 1) (mean delay, $12.8 \mathrm{msec} \pm 1.9$ $\mathrm{SD}$; variance, $3.6 ; n=25$ IPSPs each from six preparations). For individual variances, see Table 1 . The latencies of the EPSPs in MHRs and the IPSPs in ventral spinal neurons are compared in
Figure $11 B$. The increased latency and variance for the IPSPs is compatible with a disynaptic pathway from trigeminal sensory neurons, via MHR interneurons (see Discussion).

When the head skin was pressed, ventral spinal neurons that were rhythmically active during swimming received a barrage of IPSPs $(n=8)$. As has been shown previously (Boothby and Roberts, 1992b) these IPSPs were blocked by bicuculline, so are presumably GABAergic. When the head skin was pressed repeatedly the amplitude and duration of this IPSP barrage decreased $(n=4)$ (Fig. 9C), like the excitatory response in MHRs (Fig. 9B).

\section{Firing in individual MHRs stops swimming}

In seven of nine neurons tested, firing individual MHRs using positive current injection reliably stopped ongoing fictive swimming (Fig. 12). The protocol was to initiate swimming using a brief electrical stimulus to the trunk skin then inject 5-20 positive current pulses $2.4 \mathrm{sec}$ later. This gave rise to between 5 and 40 spikes in the MHR, depending on the amplitude and number of current pulses used. Two MHR neurons had no apparent effect on 


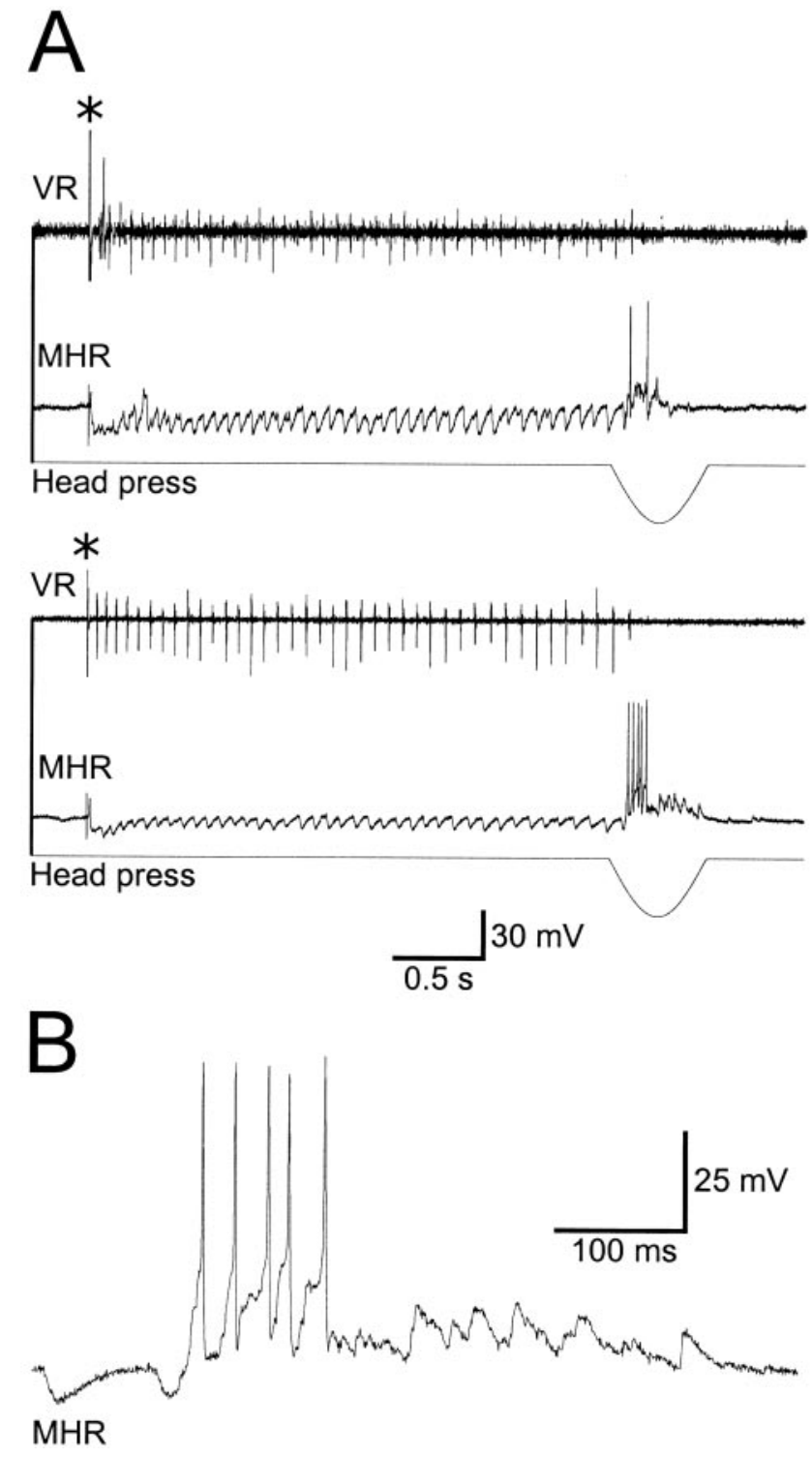

Figure 8. MHR activity during swimming and head press. A, Examples from two preparations. In each case swimming is started by a $1 \mathrm{msec}$ duration electrical stimulus to the trunk skin (at asterisk) and is monitored from the ventral roots $(V R)$. During swimming MHRs receive rhythmic, reliable IPSPs. Swimming is stopped by pressing the head skin (cement gland), when the MHRs receive excitation and fire action potentials. The anatomy of the first neuron is shown in Figure 2, $A$ and $D$, the second in Figure 2, $B$ and $E . B$, The input to the lower MHR shown at an expanded scale to clearly show individual EPSPs and spikes. swimming, however many action potentials were evoked. To assess the number of MHR impulses necessary to stop swimming, the number of MHR impulses that occurred before the last swimming cycle was counted. The number of impulses ranged from 1 to 23 (mean, $4.6 \pm 4.4$ spikes; $n=7$ tadpoles). Without such induced MHR activity, swimming always continued for many seconds before slowing and terminating spontaneously. To quantify this we counted the number of cycles before swimming stopped. Swimming continued for $239 \pm 171$ cycles $(n=15$ responses from 5 tadpoles) with no MHR spikes. After the MHRs were induced to fire swimming then continued for only $2 \pm 1$ cycles $(n=75$ responses from seven tadpoles). In these 75 responses there were 13 in which evoking MHR spikes failed to stop swimming so the stopping effect of MHR spikes was $86 \%$ reliable.

In three experiments the $\mathrm{GABA}_{\mathrm{A}}$ antagonist bicuculline (20 $\mu \mathrm{M})$ was applied to the rostral spinal cord. This had no apparent effect on fictive swimming but reversibly blocked the ability of MHR neurons to terminate swimming, even when a larger number of action potentials were evoked (Fig. 12, tested at least six times in each animal). In wash, firing individual MHRs could again reliably stop swimming when tested at least five times in each animal. This shows that the ability of MHRs to stop swimming relies on $\mathrm{GABA}_{\mathrm{A}}$-mediated inhibition, as does the normal stopping response (Boothby and Roberts, 1992a).

\section{DISCUSSION}

\section{Are reticulospinal neurons (MHRs) involved in the natural stopping pathway?}

When the head skin is pressed to stop swimming it is known that sensory neurons in the trigeminal are excited to fire action potentials (Roberts and Blight, 1975), hindbrain neurons are excited, and rhythmically active spinal neurons receive GABAergic IPSPs (Boothby and Roberts, 1992b). The simplest proposal for this stopping response is that interneurons in the hindbrain receive direct excitation from the trigeminal afferent neurons and in turn directly inhibit rhythmic spinal neurons, through the release of GABA. There are several lines of evidence that the MHRs, defined by GABA immunoreactivity by Roberts et al. (1987), are the interneurons recorded in this study.

Neurons filled in this study share many anatomical features with MHRs (see Results) and can therefore be identified with some confidence as MHRs. Roberts et al. (1987) revealed 15-20 GABA-immunopositive MHR neurons on each side of the hindbrain at stage 37-38. Many of the other reticulospinal neurons in the young Xenopus tadpole have ipsilateral descending projections (van Mier and ten Donkelaar, 1984).

The MHRs in this study were excited by pressing the cement gland, as would be expected if the MHRs are involved in the

\section{Table 1. Delays and variances (both in msec) to events after brief $(1 \mathrm{msec})$ electrical skin stimulation}

EPSPs MHRs to cement gland stimulation

\begin{tabular}{ll}
\hline Delay & Variance \\
\hline 5.97 & 0.07 \\
5.38 & 0.04 \\
5.73 & 0.15 \\
5.46 & 0.07 \\
6.45 & 0.08
\end{tabular}

IPSPs in ventral spinal neurons to cement gland stimulation

\begin{tabular}{ll}
\hline Delay & Variance \\
11.73 & 1.91 \\
12.81 & 4.15 \\
12.16 & 1.23 \\
12.11 & 1.07 \\
13.32 & 1.73 \\
14.58 & 3.85
\end{tabular}

Spikes in MHR to cement gland stimulation

\begin{tabular}{|c|c|c|c|}
\hline \multicolumn{2}{|c|}{ gland stımulatı } & \multicolumn{2}{|c|}{ skin stımulation } \\
\hline Delay & Variance & Delay & Variance \\
\hline \multirow[t]{5}{*}{13.73} & 1.70 & 9.30 & 0.06 \\
\hline & & 13.21 & 0.10 \\
\hline & & 10.13 & 0.12 \\
\hline & & 9.63 & 0.10 \\
\hline & & 9.42 & 0.09 \\
\hline
\end{tabular}

EPSPs in MHRs to tail skin stimulation 


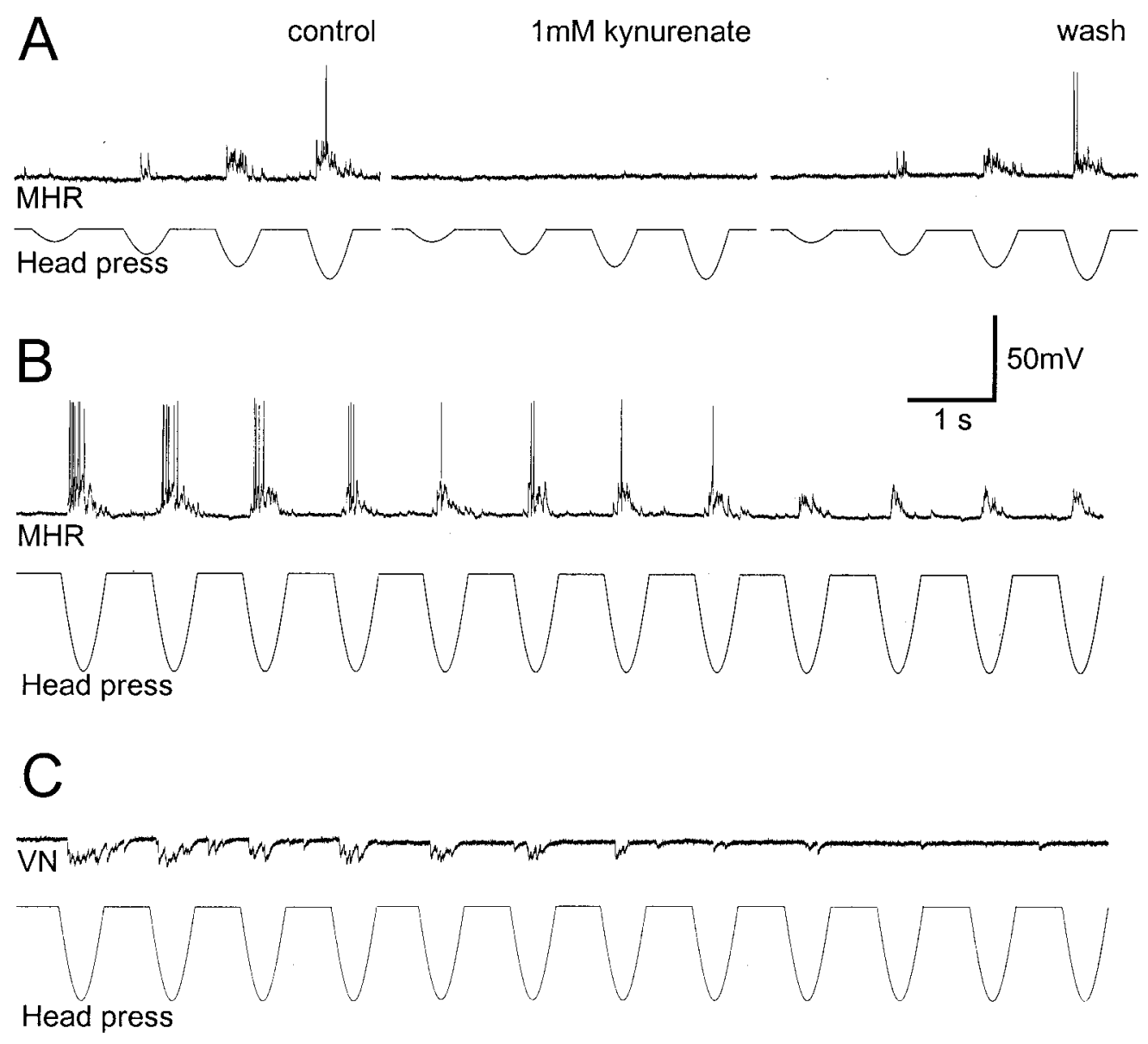

Figure 9. Responses of MHR neurons and motoneurons to pressing the head. $A$, Graded presses result in a graded barrage of EPSPs and firing at the highest intensity. This is reversibly blocked by $1 \mathrm{~mm}$ kynurenate. $B$, The excitatory response in MHR neurons reduces when presses are repeated every second. $C$, The barrage of IPSPs in a ventral, rhythmically active, neuron $(V N)$ also reduces during repeated presses.

stopping pathway. Boothby and Roberts (1992b) used extracellular recordings to show that the normal stopping response could be blocked by kynurenate, indicating that EAA-mediated excitation is important at some point in the pathway. We have shown that the excitation in MHRs was also blocked by kynurenate.

The best evidence that MHRs are involved in the normal stopping pathway is that firing in individual MHRs could reliably stop swimming. Additionally, the ability of individual MHRs to stop swimming was blocked by bicuculline, showing that it relies on the activation of $\mathrm{GABA}_{\mathrm{A}}$ receptors. The natural stopping response is also blocked by bicuculline and when the head skin is pressed GABAergic IPSPs are evoked in rhythmic spinal neurons (Boothby and Roberts, 1992b).

If most MHRs can stop swimming individually, as a population they must have a very powerful influence on the CPG. This may be to get the very reliable termination of swimming, which is seen when swimming tadpoles bumps into solid objects. Stopping is also very reliable when tested under experimental conditions by pressing on the head skin of tadpoles $(80 \%$ in restrained tadpoles, $97 \%$ in immobilized tadpoles) (Boothby and Roberts 1992a). It is quite rare to find individual neurons in vertebrates that have such a dramatic effect on behavior. The Mauthner neuron is found in the hindbrains of lampreys, sharks, teleost fish, and amphibians (Zottoli, 1978). When fired experimentally it can initiate behavior similar to a weakened escape $\mathrm{C}$-flexion in fish (for review, see
Eaton et al., 2001). Stimulating single Müller cells in the lamprey hindbrain at high frequency can also influence fictive swimming via their excitatory synapses in the spinal cord, although the results are variable (for review, see Buchanan 2001). In some cases, body or fin movements are initiated, whereas in others ongoing fictive swimming bursts can be altered in intensity or cycle period. These responses may be involved in steering. Recent work has shown that specific groups of reticulospinal neurons in the lamprey can respond to tactile stimulation by generating a plateau potential that may turn on swimming (Viana Di Prisco et al., 2000). Other reticulospinal neurons have a variety of excitatory and inhibitory effects on motoneurons firing during swimming (Zelenin et al., 2001). In the cat, reticulospinal neurons have also been shown to have inhibitory effects (Takakusaki et al., 2001). In both the lamprey and the cat the inhibition may be indirect and mediated by spinal inhibitory neurons.

The source of the midcycle IPSPs seen in MHRs during swimming is likely to be spinal commissural interneurons, which have axons projecting through the hindbrain (Yoshida et al., 1998) and are the only glycine-immunopositive neurons at this developmental stage (Dale et al., 1986). The function of these IPSPs during swimming is unclear. It is unlikely to be a phase-dependent sensory gating phenomenon (cf. Sillar and Roberts, 1988), because the stopping response is bilateral. We expect the IPSPs to lower the likelihood of activity in MHRs during swimming, 

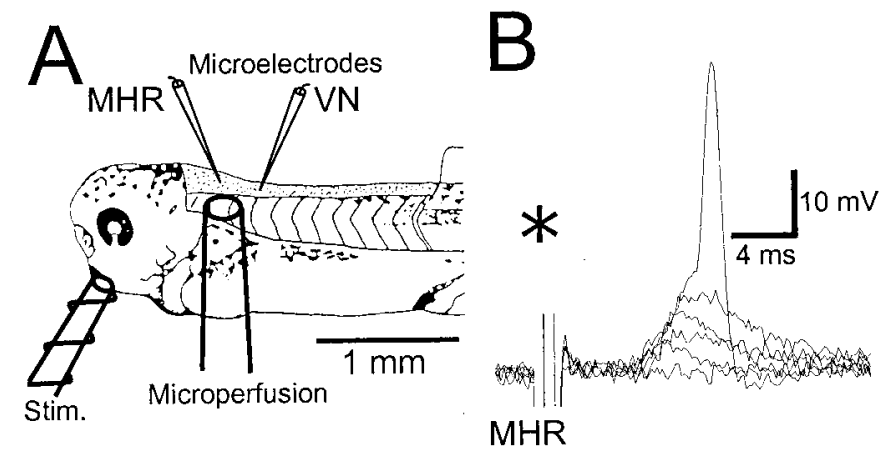

C
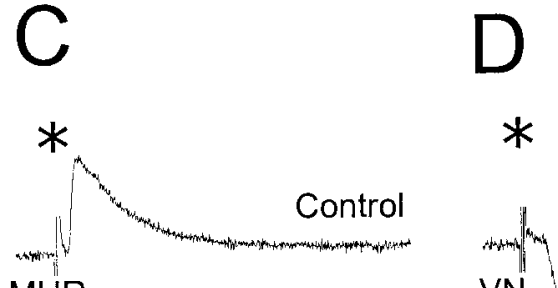

MHR
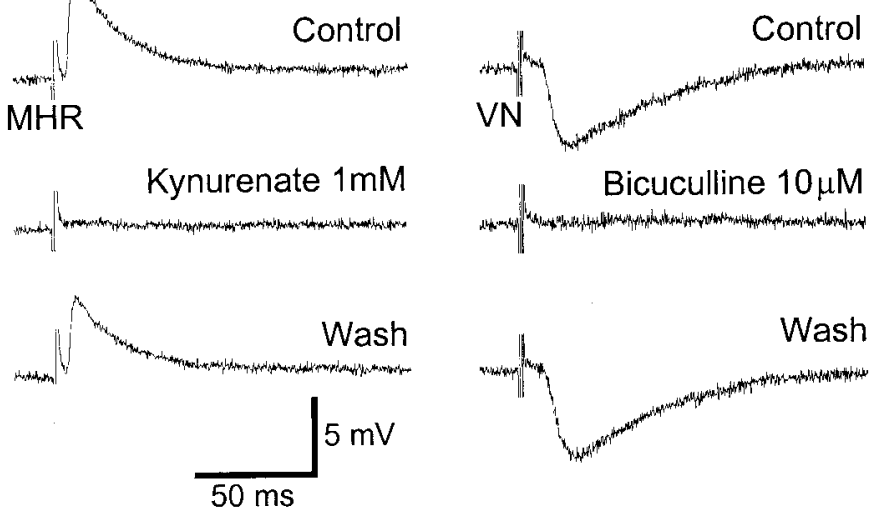

Figure 10. Responses of MHRs and ventral spinal neurons (VNs) to electrical stimulation of the cement gland. $A$, The preparation used. Microelectrodes could record from either MHRs or rostral VNs. Drugs were locally applied via a microperfusion system. $B$, As stimulus intensity is increased, the short-latency EPSP in an MHR increases in amplitude until a spike is evoked. $C$, The EPSP in MHRs is blocked by kynurenate. Each trace is an average of eight responses. $D$, The IPSP in a spinal neuron is blocked by bicuculline. Averages of eight responses. Stimulus indicated by asterisk.

although a strong enough stimulus is still able to excite the MHRs to fire impulses and therefore stop swimming. Conversely, at rest, the MHRs without IPSPs may be more easy to excite and have a higher probability of spontaneous activity. We are currently investigating the possibility that low-level activity in MHRs could decrease tadpole responsiveness during periods when they hang from a mucus strand secreted by the cement gland on the head (Lambert and Roberts, 2000).

\section{Is the stopping pathway disynaptic?}

Various possibilities have been put forward to define the stopping pathway in Xenopus, after studies involving various lesions to the CNS (Boothby and Roberts, 1992a). The short delay to, and small variance of, EPSPs in MHRs after electrical stimulation of the cement gland suggests that the connection from trigeminal sensory neurons is direct and monosynaptic. In contrast, the delay to the IPSPs in ventral spinal neurons is much longer and has a larger variance, which would result from the variable spike times in MHRs. This explanation is supported by the one case in which an MHR could be induced to spike, in which the latencies to the spike were more variable and in the same range as the IPSPs in
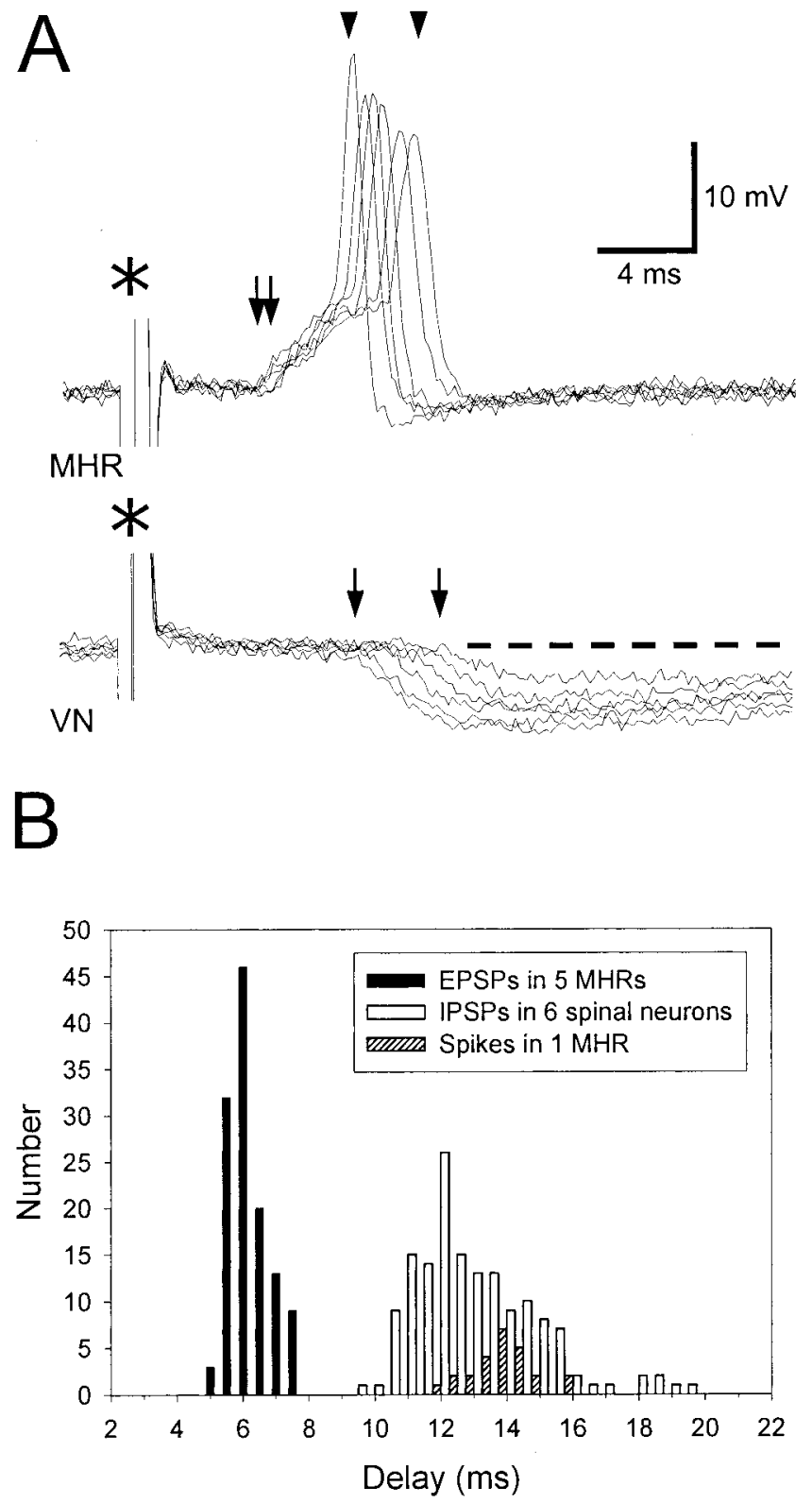

Figure 11. Latencies of responses of MHRs and VRs to electrical stimulation of the cement gland. $A$, EPSPs and spikes in an MHR (6 traces overlaid). The latency to the start of the EPSP (arrows) has a smaller variance than the latency to the spike (arrowheads). IPSPs in a ventral spinal neuron ( $\mathrm{VN}$ ) have a long and variable latency (arrows). MHR and VN recorded from two different animals. Stimulus at asterisk. $B$, Plot of latencies to EPSPs in MHRs and IPSPs in VNs shows the clear difference in timing and variability in delays. Also shown is the delay to the spike in the one MHR in which this could be evoked. Twenty-five latencies were measured in each neuron.

ventral spinal neurons (Fig. 11). The variance of $3.6 \mathrm{msec}$ in delay to the start of the IPSP is similar to that of $3.044 \mathrm{msec}$ in another possibly disynaptic pathway in the Xenopus tadpole, the contralateral EPSP evoked in motoneurons by skin stimulation (Roberts and Sillar, 1990).

The neuronal pathway that we propose for stopping swimming in Xenopus tadpoles is shown in Figure 13. Trigeminal sensory neurons innervate both the cement gland and the head skin. Slow pressure to either of these leads to multiple action potentials in a number of trigeminal afferent neurons. Their central caudally 

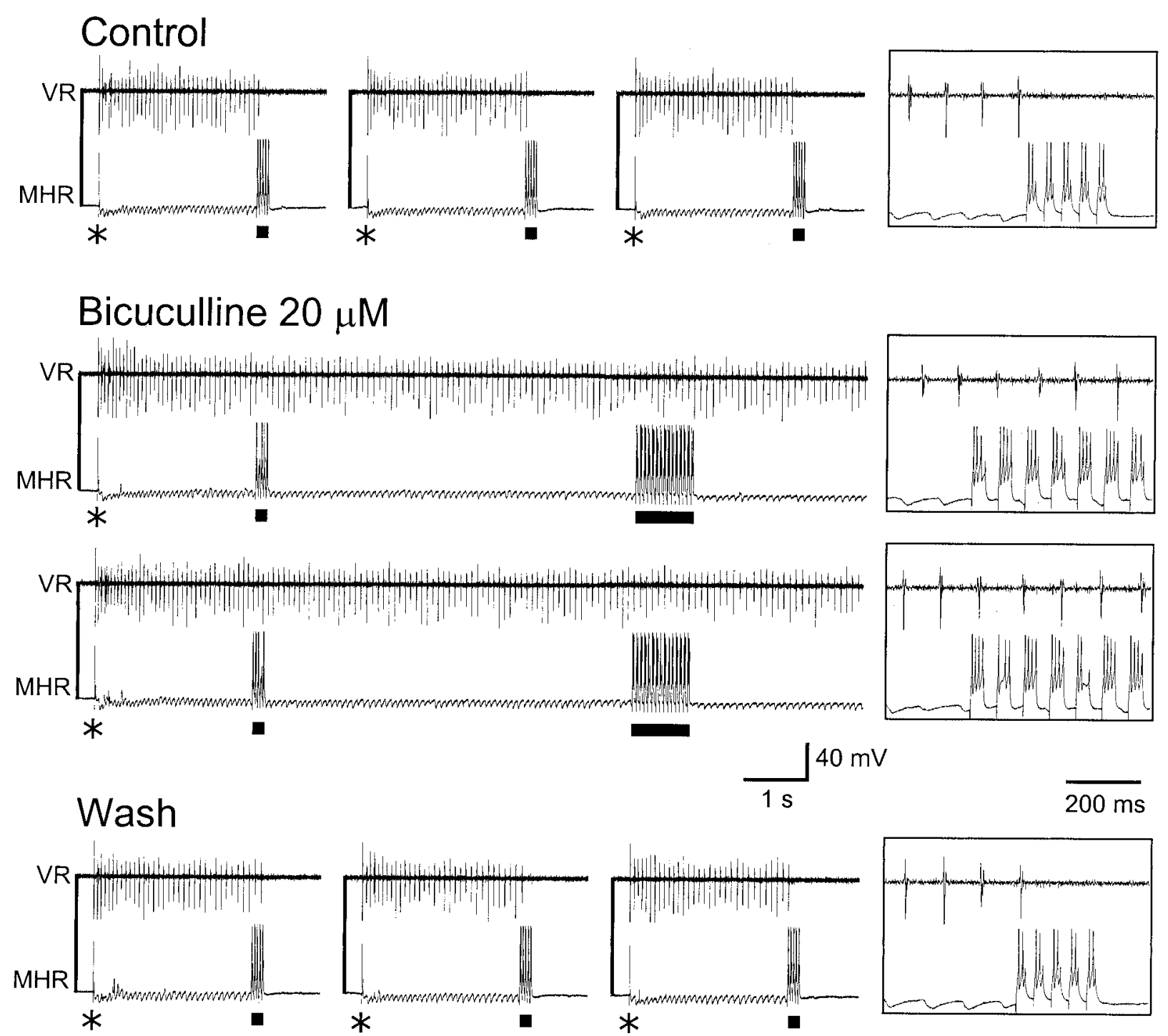

Figure 12. Stimulation of individual MHR neurons can stop ongoing swimming. In three trials in control, swimming (recorded in ventral root, $V R$ ) is started using a brief electrical stimulus to the skin (at asterisk). After $2.4 \mathrm{sec}$ five current pulses (solid bar) are injected into the MHR neuron, each resulting in two spikes (inset box shows the end of swimming at a slower time base). This stops swimming on every trial. In bicuculline, firing in the MHR neuron no longer stops swimming ( 2 trials shown). The second series of current pulses in the MHR neuron consists of 15 pulses, each giving rise to between two and four spikes (inset shows the start of this train). Swimming carried on for at least another 6 sec after end of the trace shown. After 90 sec in wash, activity in the MHR neuron can again stop swimming.

directed axons project through the hindbrain and release an EAA to excite MHR neurons. If the stimulus is sufficient, one or more MHR neurons will fire action potentials that will propagate into the spinal cord in the caudally directed axons of the MHRs. All MHRs have a commissural axon, but some also have an ipsilateral descending axon, so the action might be bilateral. The axons are presumed to make en passant synapses with neurons that they pass in the caudal hindbrain and spinal cord. At these synapses GABA is released to activate $\mathrm{GABA}_{\mathrm{A}}$ receptors and terminate swimming activity. Is this simple pathway the only one? The lesion experiments of Boothby and Roberts (1992a) suggest that there may be another commissural pathway in the rostral hindbrain that also allows trigeminal neurons to excite MHRs on the contralateral side. This would provide a second mechanism to ensure that the action of MHRs was bilateral, stopping spinal CPG neurons on both sides of the spinal cord. The delays to the start of the short-latency EPSP in MHRs supports the simplest, monosynaptic pathway. Without further evidence, for example, from paired recording from MHRs and spinal neurons, we cannot rule out less direct pathways, but at present the proposal that MHRs are excitatory and produce spinal inhibition via spinal GABAergic neurons is unlikely.

\section{Interactions with other sensory pathways}

There are four main pathways that can initiate swimming in Xenopus tadpoles (Roberts, 1998): (1) light dimming (Roberts, 1978; Jamieson and Roberts, 2000). This provided no obvious input to MHRs. (2) Skin impulses (Roberts and Stirling, 1971; Roberts 1975). Increasing skin stimulation intensity until a skin impulse was initiated produced no obvious response in MHRs. (3) Touch-sensitive receptors in the head skin. This pathway was not investigated in this study, because it is difficult to test specifically, without also stimulating receptors sensitive to pressing the head skin. (4) Touch to the trunk skin. When this pathway is 


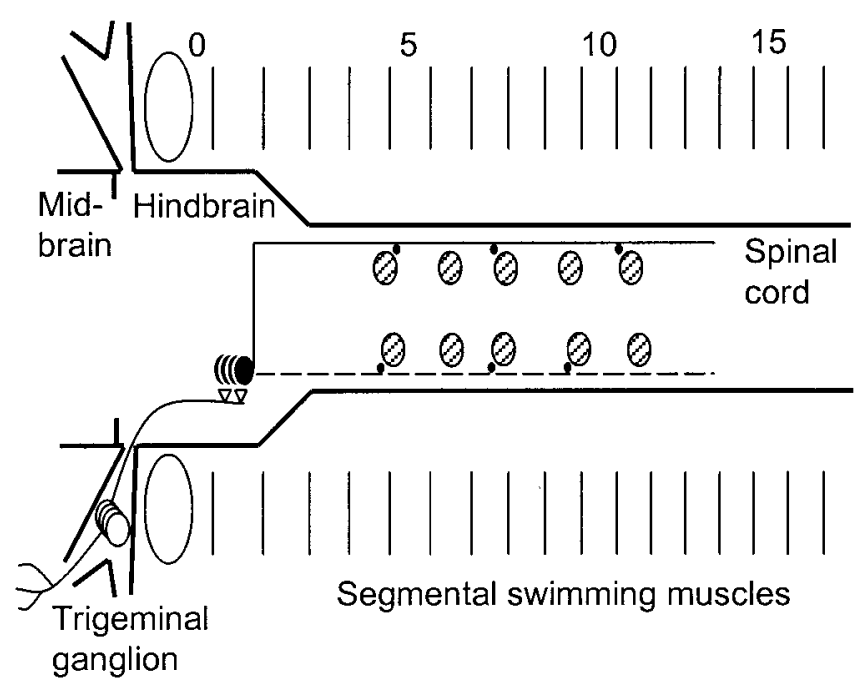

Figure 13. The proposed neuronal pathway involved in stopping swimming in Xenopus tadpoles when the head skin is pressed. This schematic diagram shows the nervous system as viewed from above (see Fig. 3). Trigeminal sensory neurons (white) have processes in the skin of the head and cement gland and an axon descending to the caudal hindbrain where synapses are made with MHRs (black). When activated the trigeminal neurons excite the MHRs by releasing an EAA. MHRs all have a contralateral descending axon (solid line), and some have an additional ipsilateral descending axon (dashed line). These axons make en passant synapses with rhythmic spinal neurons (hatching). If the stimulus intensity is sufficient, the MHRs fire action potentials that result in the release of GABA, which activates $\mathrm{GABA}_{\mathrm{A}}$ receptors and hyperpolarizes the spinal neurons to stop activity in the swimming CPG. Note that Boothby and Roberts (1992a) also proposed an additional excitatory crossing pathway in the rostral hindbrain (data not shown here).

activated, it gives rise to EPSP in MHRs. Clarke et al. (1984) calculated conduction speeds in the afferent axons from the tail skin of $0.05-0.2 \mathrm{msec}^{-1}$ and in central axons of $0.15-0.4 \mathrm{msec}^{-1}$. Because the stimulating electrode was $\sim 2 \mathrm{~mm}$ from the recording site, this would lead to theoretical conduction times of $\sim 10 \mathrm{msec}$. The latencies of these EPSPs $(\sim 11 \mathrm{msec})$ are therefore compatible with monosynaptic connections from the skin afferents. In corroboration, the EPSP latency has a low variance similar to that in other monosynaptic pathways (Roberts and Sillar, 1990). Trunk skin stimulation excites sensory neurons in the spinal cord (Rohon-Beard neurons), which have ascending and descending central axons. These axons can extend into the hindbrain (Roberts and Clarke, 1982) and are in a position to synapse with the dorsal processes of MHRs. Rohon-Beard neurons are therefore likely to be the source of the short-latency EPSPs. At higher stimulus intensities, the short-latency EPSP is followed by more variable, longer latency excitation. The source of the longer latency EPSPs and the function of either type of excitation is not clear as yet.

These inputs from the pathway that initiates swimming onto neurons involved in stopping swimming mean that the MHRs are a place where the two pathways could interact. Interactions between sensory pathways at the interneuronal level could help to explain some behavioral phenomena such as reduced responsiveness during attachment (discussed above; Lambert and Roberts, 2000).

\section{REFERENCES}

Boothby KM, Roberts A (1992a) The stopping response of Xenopus laevis tadpoles: behavior, development and physiology. J Comp Physiol [A] 170:171-180.

Boothby KM, Roberts A (1992b) The stopping response of Xenopus laevis tadpoles: pharmacology and intracellular physiology of rhythmic spinal neurones and hindbrain neurones. J Exp Biol 169:65-86.

Buchanan JT (2001) Contributions of identifiable neurons and neuron classes to lamprey vertebrate neurobiology. Prog Neurobiol 63:441-466.

Clarke JDW, Hayes BP, Hunt SP, Roberts A (1984) Sensory physiology, anatomy and immunohistochemistry of Rohon-Beard neurones in embryos of Xenopus laevis. J Physiol (Lond) 348:511-525.

Dale N, Ottersen OP, Roberts A, Storm-Mathisen J (1986) Inhibitory neurones of a motor pattern generator in Xenopus revealed by antibodies to glycine. Nature 324:255-257.

Eaton RC, Lee RKK, Foreman MB (2001) The Mauthner cell and other identified neurons of the brainstem escape network of fish. Prog Neurobiol 63:467-485.

Foster RG, Roberts A (1982) the pineal eye in Xenopus laevis embryos and larvae: a photoreceptor with a direct excitatory effect on behavior. J Comp Physiol [A] 145:413-419.

Fraenkel G (1932) Untersuchungen über die koordination von reflexen und automatisch-nervösen rhythmen bei insekten. I. Die flungreflexe der insecten und ihre koordination. Z Vergl Physiol 16:371-393.

Gray J, Sand A (1936) The locomotory rhythm of the dogfish. J Exp Biol 13:200-209.

Hayes BP, Roberts A (1983) The anatomy of two functional types of mechanoreceptive "free" nerve ending in the head skin of Xenopus tadpoles. Proc R Soc Lond B Biol Sci 218:61-76.

Holstege JC (1991) Ultrastructural evidence for GABAergic brainstem projections to spinal motoneurons in the rat. J Neurosci 11:159-167.

Jamieson D, Roberts A (2000) Responses of young Xenopus laevis tadpoles to light dimming: possible roles for the pineal eye. J Exp Biol 203:1857-1867.

Lambert TD, Roberts A (2000) Tonic activity of trigeminal sensory neurons and reduced responsiveness during cement gland attachment in hatchling Xenopus laevis tadpoles. J Physiol (Lond) 523:272.P.

Li W-C, Perrins R, Soffe SR, Yoshida M, Walford A, Roberts A (2001) Discriminating classes of spinal interneuron in Xenopus tadpoles. J Comp Neurol 441:248-265.

Llinas R, Terzuolo CA (1964) Mechanisms of supraspinal actions upon spinal cord activities. Reticular inhibitory mechanisms on alphaextensor motoneurons. J Neurophysiol 27:579-591.

Magoun HW, Rhines R (1946) An inhibitory mechanism in the bulbar reticular formation. J Neurophysiol 9:165-171.

Mori S (1987) Integration of posture and locomotion in acute decerebrate cats and in awake, freely moving cats. Prog Neurobiol 28:161-196.

Nieuwkoop PD, Faber J (1956) Normal tables of Xenopus laevis (Daudin). Amsterdam: North-Holland.

Pringle JWS (1974) Locomotion: flight. In: The physiology of the Insecta, Vol 3 (Rockstein M, ed), pp 433-476. London: Academic.

Roberts A (1975) Mechanisms for the excitation of "free nerve endings". Nature 253:737-738.

Roberts A (1978) Pineal eye and behavior in Xenopus tadpoles. Nature 273:774-775.

Roberts A (1980) The function and role of two types of mechanoreceptive "free" nerve endings in the head skin of amphibian tadpoles. J Comp Physiol [A] 135: 341-348.

Roberts A (1998) Skin sensory systems of amphibian embryos and young larvae. In: Amphibian biology, Vol 3, "Sensory perception" (Heatwole H, ed), pp 923-935. Chipping Norton, NSW, Australia: Surrey Beatty \& Sons.

Roberts A, Blight AR (1975) Anatomy, physiology and behavioural role of sensory nerve endings in the cement gland of embryonic Xenopus. Proc R Soc Lond B Biol Sci 192:111-127.

Roberts A, Clarke JDW (1982) The neuroanatomy of an amphibian spinal cord. Philos Trans R Soc Lond B Biol Sci 296:195-212.

Roberts A, Sillar KT (1990) Characterization and function of spinal excitatory interneurons with commissural projections in Xenopus laevis tadpoles. Eur J Neurosci 2:1051-1062.

Roberts A, Stirling CA (1971) The properties and propagation of a cardiac-like impulse in the skin of young tadpoles. Z Vergl Physiol 71:295-310.

Roberts A, Dale N, Ottersen OP, Storm-Mathisen J (1987) The early development of neurons with GABA immunoreactivity in the CNS of Xenopus laevis tadpoles. J Comp Neurol 261:435-449.

Roberts A, Soffe SR, Perrins R (1997) Spinal Networks controlling swimming in hatchling Xenopus tadpoles. In: Neurons, networks and motor behavior. (Stein PSG, Grillner S, Selverton AI, Stuart DG, eds) Cambridge, MA: MIT.

Roberts A, Hill NA, Hicks R (2000) Simple mechanisms organise orientation of escape swimming in embryos and hatchling tadpoles of Xenopus laevis. J Exp Biol 203:69-85.

Satterlie RA, LaBarbera M, Spencer AN (1985) Swimming in the pterpod mollusc, Clione limacina. I. Behavior and morphology. J Exp Biol 166:189-204.

Sillar KT, Roberts A (1988) A neuronal mechanism for sensory gating during locomotion in a vertebrate. Nature 331:262-265.

Sillar KT, Roberts A (1992) Phase-dependent modulation of a cutane- 
ous sensory pathway by glycinergic inhibition from the locomotor rhythm generator in Xenopus embryos. Eur J Neurosci 4:1022-1034.

Soffe SR (1990) Active and passive membrane properties of spinal cord neurons which are rhythmically active during swimming in Xenopus embryos. Eur J Neurosci 2:1-10.

Soffe SR, Perrins R (1997) Neuronal firing properties and swimming motor patterns in four amphibian embryos: Xenopus, Rana, Bufo and Triturus. J Comp Physiol 181:71-81.

Stein PSG, Grillner S, Selverston AI, Stuart DG (1997) Neurons, networks and motor behavior. Cambridge, MA: MIT.

Takakusaki K, Kohyama J, Matsuyama K, Mori S (2001) Medullary reticulospinal tract mediating generalised motor inhibition in cats: parallel inhibitory mechanisms acting on motoneurons and on interneuronal transmission in reflex pathways. Neuroscience 103:511-527.

van Mier P, ten Donkelaar HJ. (1984) Early development of descending pathways from the brain stem to the spinal cord in Xenopus laevis. Anat Embryol 170:295-306.

Viana Di Prisco G, Pearlstein E, Le Ray D, Robitaille R, Dubuc R (2000) A cellular mechanism for the transformation of a sensory input into a motor command. J Neurosci 20:8169-8176.

Yoshida M, Roberts A, Soffe SR (1998) Axon projections of reciprocal inhibitory interneurons in the spinal cord of young Xenopus tadpoles and implications for the pattern of inhibition during swimming and struggling. J Comp Neurol 400:504-518.

Zelenin PV, Grillner S, Orlovsky GN, Deliagina TG (2001) Heterogeniety of the population of command neurons in the lamprey. J Neurosci 21:7793-7803.

Zottoli SJ (1978) Comparative morphology of the Mauthner cell in fish and amphibians. In: Neurobiology of the Mauthner cell (Faber DS, Korn H, eds), pp 13-45. New York: Raven. 